\title{
Reconstrucciones "Resilientes” de la Identidad Profesional del Profesorado: Endoprivatización y Cultura Performativa en Andalucía (España)
}

\author{
"Resilient" Reconstructions of Teachers Professional Identity: \\ Endoprivatization and Performative Culture in Andalusia \\ (Spain)
}

\author{
Javier Molina-Pérez * \\ Julián Luengo \\ Universidad de Granada, España
}

\begin{abstract}
La investigación tiene como objeto de estudio comprender cómo las reformas promulgadas bajo la agenda neoliberal podrían estar transformando la identidad del profesorado y las prácticas docentes. Metodológicamente se sigue un enfoque inductivo concretado en la propuesta de la Teoría Fundamentada. Se define un muestreo teórico determinado por criterios de relevancia y acotado a la Comunidad Autónoma de Andalucía. Se plantea una entrevista semiestructurada en profundidad, desarrollada con doce docentes de Educación Secundaria Obligatoria. Los resultados evidencian la configuración de un profesor performativo, limitado pedagógicamente, deslegitimado por la administración y ajeno a cualquier espacio de decisión sobre su tarea en el aula. Por ello, la agenda neoliberal reclama una nueva reconstrucción "resiliente" de la identidad profesional. Se pretende soslayar el sentimiento de hastío y vulneración intelectual que percibe el profesorado generando nuevos perfiles profesionales asociados a un profesor flexible, ágil y emocionalmente inteligente. Se trata de reducir los espacios críticos a la esfera individual y ser capaz de encontrar equilibrios emocionales que permitan a los profesionales insatisfechos hacer frente a las presiones del contexto neoliberal.
\end{abstract}

Descriptores: Neoliberalismo; Identidad; Docente de secundaria; Política educacional; Reforma de la educación.

The purpose of this research is to understand how the reforms performed under the neoliberal agenda could be transforming the identity of teachers together with the teaching practices. Methodologically, an inductive approach has been followed, stablished in the Grounded Theory. A theoretical sampling is defined by relevant criteria and limited to the Autonomous Community of Andalusia. An in-depth semistructured interview is proposed; it's been developed with twelve teachers of Compulsory Secondary Education. The results show the shape of a performative teacher, who is pedagogically limited, not considered in spaces of decision about their performance in the classroom, and whose role is delegitimized by the administration. Therefore, the neoliberal agenda calls for a new "resilient" reconstruction of professional identity. The aim is to put aside that feeling of weariness and intellectual blaming that teachers perceive producing new professional profiles typical of a flexible, agile and emotionally intelligent teacher. It is about narrowing critical spaces down to the individual sphere and being able to find emotional balances that will allow dissatisfied professionals to deal with the pressures of the neoliberal context.

Keywords: Neoliberalism; Identity; Secondary school teachers; Educational policy; Educational reform.

*Contacto: javimp@ugr.es

Recibido: $\quad 11$ de octubre 2019

ISSN: 1696-4713

$1^{\text {a }}$ Evaluación: 16 de enero 2020

www.rinace.net/reice/

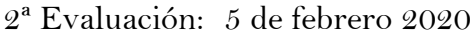

revistas.uam.es/reice

Aceptado: 17 de febrero 2020 


\section{Revisión de la literatura}

El favorecimiento de la competitividad entre centros, los modelos gerenciales y los mecanismos de rendición de cuentas, como principales prácticas de las reformas educativas neoliberales, están acelerando los estudios que se centran en explorar las posibles tensiones y conflictos que experimentan los docentes. $\mathrm{Y}$ es que, de acuerdo con la investigación de Day (2002, p. 683), "las reformas tienen un impacto sobre las identidades del profesorado". Este conjunto de prácticas políticas ha sido categorizado por Ball y Youdell (2008) como dinámicas de privatización endógena o endoprivatización, destacando, por su amplio análisis en el contexto español, las políticas de cuasimercado, la agenda reformista de la Nueva Gestión Pública (NGP) y las medidas de rendición de cuentas. De este modo, la investigación sobre las prácticas de cuasimercado educativo evidencia unos procesos donde los centros y su profesorado se esfuerzan para mejorar los resultados académicos en evaluaciones constantes de rendimiento para competir y atraer a "clientes" (Luengo y Molina-Pérez, 2018). Especialmente significativa es la producción académica que se ocupa de estudiar las reformas basadas en la agenda de la NGP, fundamentadas en parámetros empresariales, que acercan los modelos funcionales del sector privado al sector público (Verger y Normand, 2015). Por último, la cultura auditora o performativa que se ha extendido en los sistemas educativos ocupa un amplio espacio en los estudios de política educativa. Bajo la noción de performatividad se hace referencia a "una tecnología, una cultura y una modalidad de reglamentación que utiliza evaluaciones, comparaciones e indicadores como medios para controlar, desgastar y producir cambio" (Ball, 2003, p. 89).

En consecuencia, el objeto de estudio se centra en el conjunto de reformas de corte neoliberal que promueven procesos de endoprivatización y que podrían estar transformando la identidad profesional del profesorado y la diversidad de prácticas que conforman la profesionalidad docente. En este sentido, se señala que se entiende la identidad profesional desde una posición interaccionista con la práctica docente. Así lo han entendido trabajos como el de Bolívar, Domingo y Pérez-García (2014), considerando las categorías de "identidad profesional" y "profesionalidad docente" como dos caras de la misma moneda. Mientras que la identidad profesional docente se vincula a una dimensión más subjetiva, asociada a vivencias individuales y percepciones sociales; la profesionalidad docente abarca patrones relacionados con los conocimientos, las competencias y los rasgos que constituyen el quehacer docente. Por ello, como ha estudiado Hendrikx (2019), hacer interaccionar la identidad atribuida por el propio docente desde dimensiones subjetivas con su práctica cotidiana en el aula, condicionada por sus conocimientos, competencias y rituales profesionales, puede dar cuenta de desequilibrios identitarios, crisis de identidad o nuevas fabricaciones identitarias que se desarrollan para ser eficaz en los contextos de trabajo actuales (Courtney y Gunter, 2019). En esta línea de trabajo, Day y Gu (2015, p. 52) hacen referencia a que actualmente existe "una mayor necesidad por parte de los profesores de contar con la capacidad de ser resilientes".

El constructo de resiliencia ha evolucionado desde sus orígenes en el campo de la psiquiatría y la psicología del desarrollo, donde se definía en torno a una serie de características personales que posibilitaban la adaptación y el progreso de los niños en riesgo de experimentar circunstancias negativas para su vida (Waller, 2001). La década de los ochenta del siglo anterior supuso un nuevo paradigma en el estudio de la resiliencia. Pasó a concebirse como un complejo relativo, dinámico y en constante evolución que se 
focalizaba en la adaptación positiva y el desarrollo de las personas ante vicisitudes y desafíos cotidianos (Luthar, Cicchetti y Becker, 2000). En educación se están sucediendo los estudios sobre la importancia de incorporar la resiliencia en el trabajo del profesor y en el desarrollo de su identidad. Se argumenta que ya no tiene que estar ceñida a reponerse de experiencias traumáticas y se asocia con una cualidad positiva que permite tener la "capacidad para mantener el equilibrio y el sentido del compromiso y de la pertenencia en los distintos universos cotidianos" (Gu y Day, 2013, p. 45).

La noción de identidad es compleja puesto que no puede objetivarse ni definirse en términos absolutos porque se compone de categorías sociales y psicológicas diversas y difusas que establecen relaciones simbióticas. Una aproximación conceptual ampliamente compartida es la de Dubar (2000, p. 109), entendiéndola como "el resultado a la vez estable y provisional, individual y colectivo, subjetivo y objetivo, biográfico y estructural, de los diversos procesos de socialización que, conjuntamente, construyen los individuos y definen las instituciones". Esto implica el rechazo de interpretaciones lineales que no consideren el contexto de desarrollo personal y profesional para incorporar dimensiones que asuman su carácter complejo, dinámico, biográfico y contextualizado (Garner y Kaplan, 2019). De la misma forma, la noción de identidad profesional, pese a ser ampliamente estudiada, es frecuentemente inoperativa y no se encuentra un consenso sobre lo que implica o una orientación compartida sobre cómo abordar su estudio (Hendrikx, 2019). Ante esta cuestión, conviene explicitar que esta investigación parte de la tesis de que no existe una identidad profesional unificada, sino formas identitarias múltiples en procesos permanentes de (re)construcción. En coherencia con esta idea, se considera que la identidad profesional atiende a un proceso de autoconstrucción que integra aspectos biológicos, biográficos y sociales en un ejercicio permanente de negociación con las demandas políticas, los compañeros en el lugar de trabajo, las estructuras y relaciones de poder que se dan en los distintos espacios de actuación y la interacción con el alumnado y las familias (Sancho-Gil et al., 2014). Por consiguiente, se considera que la identidad profesional se elabora a lo largo de la vida del profesor y está vinculada a etapas y epifanías, en un espacio donde interaccionan factores biográficoemocionales, factores relacionados con el contexto escolar y factores vinculados al entramado político (Day, 2018).

Este cuerpo teórico-conceptual se concreta, compartiendo las aportaciones de Bolívar (2006) y posteriormente Bolívar y Domingo (2019), en un conjunto de dimensiones que conforman y definen la identidad profesional. Así, se habla de identidad profesional del profesorado para referirse a aspectos relacionados con la autoimagen, que interpelan al significado que el propio profesor atribuye a su yo profesional; el reconocimiento social percibido por el profesorado, destacando las figuras administrativas relevantes, la política educativa o la relación con familias y alumnado; el grado de satisfacción, entendido como una reacción afectivo-emocional concerniente al desarrollo de un trabajo y determinada por el (des)equilibrio entre la situación profesional actual y la expectativa esperada, merecida o deseada; las relaciones sociales en el centro, que puedan comprender el apoyo y reconocimiento interno; la actitud ante el cambio en forma de adaptación o resistencia a las reformas y a la cultura escolar; las competencias profesionales, ligadas a la autonomía pedagógica percibida; y las expectativas de futuro de la profesión y en la profesión.

Para hacer operativo su estudio se parte de un posicionamiento que entiende que la identidad se proyecta mediante la narrativa, en tanto que, como un autorrelato, no es solo un recuerdo de una trayectoria pasada sino un modo de negociar la historia personal para 
dar sentido al presente (Bolívar, 2006). Este acercamiento conlleva una elección metodológica que se sirve de los trabajos que centran su atención sobre el valor del relato como medio para construir la identidad (profesional). El sujeto, como expone Ricoeur (1996),

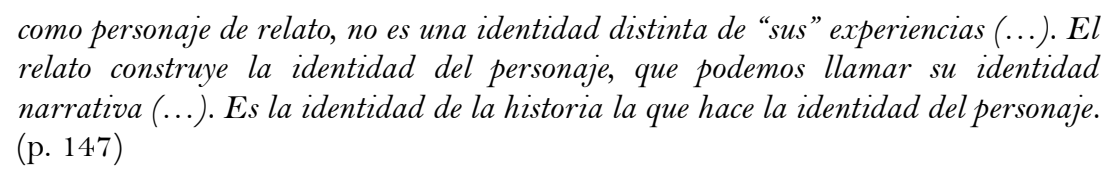

En esta línea, siguiendo los trabajos de Bolívar y Domingo (2019) y Courtney y Gunter (2019), se considera que la narrativa constituye al sujeto, más que expresarlo o representarlo. La narración actúa como una transacción que media entre los sucesos vividos, la historia del presente y la expectativa del futuro.

En consecuencia, una investigación comprometida con la transformación y la mejora de la educación requiere de un ejercicio pausado de comprensión e interacción con uno de sus principales agentes: el profesorado. Por ello, se plantean las siguientes preguntas de investigación que serán abordadas en este artículo: ¿están transformando las prácticas de endoprivatización la identidad profesional del profesorado? ¿Cómo describe el profesorado su práctica docente en el marco de las reformas políticas de endoprivatización? ¿Qué tipo de cualidades, prácticas y competencias demanda la agenda neoliberal al profesorado? De este modo, el objetivo general del trabajo responde a:

- Describir y comprender de qué forma las políticas de endoprivatización pueden transformar la identidad profesional docente y las prácticas que fundamentan su profesionalidad desde el análisis de narrativas del profesorado.

Este objetivo general se concreta en los siguientes objetivos específicos:

- Entender la incidencia de las dinámicas de endoprivatización en la reconstrucción de la identidad profesional docente.

- Estudiar la influencia de las políticas educativas que promueven procesos de endoprivatización en el desarrollo de prácticas docentes.

\section{Método}

Este trabajo prioriza las narrativas de los participantes, por lo que se sigue un enfoque metodológico cualitativo para la recogida y el tratamiento de la información. Por ello, no se plantean hipótesis apriorísticas o relaciones causales entre variables y se opta por establecer preguntas de investigación que favorecen marcos inductivos de comprensión hermenéutica (Smith, Flower y Larkin, 2009). En efecto, la investigación se desarrolla bajo un marco interpretativo en un nivel aprehensivo-analítico. A su vez, se concreta en un método inductivo, indicado para desarrollar la propuesta de la Teoría Fundamentada como base para la construcción de una teoría formal emergente a partir de las teorías sustantivas de los participantes (Glaser y Strauss, 1967). Se opta por la Teoría Fundamentada como una mirada interpretativa de las informaciones recabadas de los participantes que invita a desarrollar un sentido narrativo al conjunto de informaciones emergentes (Charmaz, 2004). 


\subsection{Instrumento de obtención de la información}

Se utiliza la entrevista cualitativa como técnica de recogida de información con una finalidad hipotético-inductiva (Wengraf, 2012). Concretamente, se desarrolla una entrevista semiestructurada en profundidad para seguir los procesos inductivos propios de la Teoría Fundamentada. Se adopta este modelo de entrevista atendiendo a distintos criterios. Primero, para ajustarse a una serie de bloques temáticos que, considerando las bases epistemológicas expresadas con anterioridad, entienden la identidad como un constructo en el que intervienen factores biográfico-emocionales, factores relacionados con el espacio de trabajo y factores relativos al contexto sociocultural y político. Segundo, por ser la técnica que permite compilar amplias unidades de significado atendiendo a objetivos de investigación muy concretos. Por último, por facilitar un acercamiento al informante que favorece un espacio dialógico donde se aportan claves que indagan en las dimensiones éticas, personales y profesionales que están relacionadas con los objetivos propuestos (Kvale, 2015). Así mismo, la modalidad de entrevista utilizada, conforme a la singularidad del enfoque cualitativo del trabajo, aporta claves de carácter ideográfico. En consecuencia, las narrativas que se exponen en esta investigación no pretenden ser generalizables o explicar la realidad de otros contextos (Denzin y Lincoln, 2012).

Es preciso señalar que las cuestiones de la entrevista se plantean de forma abierta y flexible, siguiendo un modelo laxo, poco directivo y sin priorizar los bloques que la componen para favorecer los espacios narrativos de interés que muestran los participantes. En los bloques aludidos, destacaron especialmente dimensiones de la identidad como la "autoimagen", partiendo de cuestiones como: ¿piensa que es bueno(a) en lo que hace, incluso, si los indicadores o estándares dicen lo contrario? El "grado de satisfacción" o el "reconocimiento social percibido", desde propuestas como: ¿las expectativas previas sobre su trabajo se corresponden con su situación actual? ¿Hay algún sentir mayoritario en el profesorado referente a los cambios que pueden estar generando las políticas educativas? Las "competencias profesionales" y su concepción sobre la autonomía profesional implicaron respuestas elaboradas sobre asuntos como: ¿qué incidencia tienen las evaluaciones estandarizadas en su práctica? ¿Cómo describiría al docente competente en el contexto actual? Y, por último, la dimensión "actitud ante el cambio" desde el marco de cuestiones como: ¿ha experimentado alguna situación profesional compleja en los últimos años? Si es así, ¿cómo está negociando esos desajustes profesionales?

\subsection{Participantes}

La selección de los participantes en la investigación se realiza atendiendo a la técnica snowball sampling (Noy, 2008), en base a los siguientes criterios:

- Profesorado que desarrolle su práctica en centros de titularidad y gestión pública.

- Profesorado que no ocupe actualmente el cargo de dirección.

- Profesorado que, no siendo directores o directoras, tengan o hayan tenido alguna función directiva.

- Profesorado de distintas áreas de conocimiento.

- Profesorado cuyo trabajo se desarrolle en centros de diversa ubicación geográfica. 
- Profesorado de centros con diferente Índice Socio-Económico y Cultural (ISC $\left.{ }^{1}\right)$.

Los criterios se definen con la intención de considerar perfiles docentes con diferentes experiencias y situaciones profesionales. En esta fase, se busca aumentar el volumen de las informaciones, su complejidad, su diversidad y su interactividad. Sin embargo, no se pretende que las diferentes trayectorias o condiciones del profesorado sirvan como variables para desarrollar análisis diferenciados. En línea con otras investigaciones del ámbito (véase Bolívar, Domingo y Pérez-García (2014)), la finalidad de este procedimiento radica en relacionar las narrativas de los participantes con diversas características profesionales para hacer emerger un espacio teórico que pueda dar cuenta de la identidad profesional de los docentes para el contex to de estudio.

Para acceder al profesorado se contacta con nueve directores ${ }^{2}$ de Institutos de Educación Secundaria (IES) de la Comunidad Autónoma de Andalucía (España). Los directores de los IES se consideran informantes clave por ser responsables de los espacios institucionales a los que se accede y por el conocimiento que presentan sobre las particularidades profesionales del profesorado. De esta forma, se asegura el cumplimiento de los criterios a, e y f por parte de los investigadores. A los directores se les solicita, siguiendo el criterio de relevancia de caso indicado en la técnica, acceder al profesorado que, atendiendo a su disponibilidad y disposición, pueda cumplir con los requerimientos b, c y d. Se trata de un muestreo teórico, donde la cantidad de informantes no está definida de antemano (Flick, 2014). Así, son doce los participantes que forman parte de esta fase de la investigación. Las doce entrevistas han sido grabadas en audio y transcritas. Este número se determina atendiendo al criterio de saturación teórica, expuesto por Glaser y Strauss (1967), referente a un estado del trabajo de campo donde nuevas narrativas no añaden aspectos relevantes a los objetivos planteados. En esta línea, siguiendo las aportaciones de Vallés (2009) para la técnica empleada en esta investigación, se da por finalizado el proceso simultáneo de análisis y recolección de informaciones cuando "nuevas entrevistas no añaden nada relevante a lo conocido" (p. 68) y se reiteran las narrativas que componen las categorías existentes.

En el apartado de resultados se emplean extractos narrativos de los participantes utilizando códigos para su identificación ${ }^{3}$, al objeto de favorecer un encuentro dialéctico entre la realidad percibida por el profesorado, los hallazgos científicos y las interpretaciones de los investigadores. En el cuadro 1 se detallan algunas características profesionales de los participantes para favorecer la contextualización de sus manifestaciones.

\footnotetext{
${ }^{1}$ El ISC es una variable que clasifica al alumnado de los centros según las condiciones socioeconómicas y culturales de sus familias. Se determina por medio de un cuestionario que mide aspectos referentes al nivel cultural, económico y los recursos disponibles para el estudio.

2 Aunque las direcciones educativas no son informantes de esta etapa de la investigación, el acceso al profesorado entrevistado, que no ocupa el puesto de dirección, pudo realizarse gracias al contacto facilitado por los directores de los centros. Previamente se les informó de la investigación vía email (con documentos informativos, conocimiento informado y compromiso ético) y, posteriormente, vía telefónica.

${ }^{3}$ Siguiendo las consideraciones éticas del Institutional Review Boards (IRB) se omite el nombre de los participantes y los informantes conocen las finalidades de la investigación. Su participación ha estado sujeta a posibles abandonos y han contrastado las narrativas antes de su publicación.
} 
Cuadro 1. Códigos asignados a los participantes e informaciones contextuales

\begin{tabular}{|c|c|}
\hline PARTICIPANTES & INFORMACIONES CONTEXTUALES \\
\hline Dmc 1 & $\begin{array}{l}\text { Licenciada en Matemáticas. Amplia experiencia en el sistema educativo. Sin } \\
\text { funciones directivas. IES de ISC medio-alto en el centro de la ciudad. }\end{array}$ \\
\hline Dmzr2 & $\begin{array}{l}\text { Licenciado en Matemáticas. Amplia experiencia en el sistema educativo. Sin } \\
\text { funciones directivas. IES de ISC medio-bajo en zona rural. }\end{array}$ \\
\hline Dhpe3 & $\begin{array}{l}\text { Licenciada en Humanidades. Corta experiencia en el sistema educativo. Sin } \\
\text { funciones directivas. IES de ISC medio en barrio periférico de ciudad. }\end{array}$ \\
\hline Dmpe4 & $\begin{array}{l}\text { Licenciada en Matemáticas. Amplia experiencia en el sistema educativo. } \\
\text { Actualmente es vicedirectora. IES de ISC medio en barrio periférico de } \\
\text { ciudad. }\end{array}$ \\
\hline Dhzr 5 & $\begin{array}{l}\text { Licenciado en Historia. Amplia experiencia en el sistema educativo. Ha } \\
\text { ocupado distintos cargos directivos. IES de ISC medio-bajo en zona rural. }\end{array}$ \\
\hline Dfc6 & $\begin{array}{l}\text { Licenciado en Filosofía. Amplia experiencia en el sistema educativo. Sin } \\
\text { funciones directivas. IES de ISC medio-alto en el centro de la ciudad. }\end{array}$ \\
\hline Dmtpe7 & $\begin{array}{l}\text { Licenciada en Matemáticas. Amplia experiencia en el sistema educativo. Sin } \\
\text { funciones directivas. IES de ISC medio en barrio periférico de ciudad. }\end{array}$ \\
\hline $\operatorname{Dgc} 8$ & $\begin{array}{l}\text { Licenciada en Geografía. Amplia experiencia en el sistema educativo. Sin } \\
\text { funciones directivas. IES de ISC medio-alto en el centro de la ciudad. }\end{array}$ \\
\hline Dtszr9 & $\begin{array}{l}\text { Licenciada en Trabajo Social. Amplia experiencia en el sistema educativo. } \\
\text { Sin funciones directivas. IES de ISC medio-bajo en zona rural. }\end{array}$ \\
\hline Defc 10 & $\begin{array}{l}\text { Licenciado en Educación Física. Amplia experiencia en el sistema educativo. } \\
\text { Ocupa el cargo de jefe de estudios. IES de ISC medio-alto en el centro de la } \\
\text { ciudad. }\end{array}$ \\
\hline Dfic 11 & $\begin{array}{l}\text { Licenciado en Filología Inglesa. Amplia experiencia en el sistema educativo. } \\
\text { Sin funciones directivas. IES de ISC medio-alto en el centro de la ciudad. }\end{array}$ \\
\hline Dmgzr 12 & $\begin{array}{l}\text { Licenciada en Matemáticas. Amplia experiencia en el sistema educativo. Ha } \\
\text { ocupado cargos de gestión. IES de ISC medio-bajo en zona rural. }\end{array}$ \\
\hline
\end{tabular}

Fuente: Elaboración propia.

\subsection{Análisis de datos y categorías de significado}

Para el análisis de datos se realiza una codificación inductiva de las informaciones recabadas optando por ajustarse con fidelidad al discurso de los participantes, entendido como unidad de significado, que deviene en la conformación de pre-categorías. La indagación posterior profundiza en el establecimiento de relaciones entre los contenidos narrativos codificados en las unidades de significado para favorecer un proceso emergente de integración de nodos (Strauss y Corbin, 2002). Con este fin, se utiliza el software cualitativo QSR NVivo12 y se ejecuta un análisis de conglomerados del que se obtiene un diagrama (figura 1) que asocia unidades de significado que presentan coincidencias en el contenido de codificación.

El resultado obtenido comprende un proceso más amplio de organización y reorganización de la información atendiendo a similitudes, coincidencias, emparejamientos y saturación. Para ello, se sigue el procedimiento sistemático que establece la Teoría Fundamentada y que comprende la codificación viva de las informaciones, la codificación axial y la codificación selectiva. En el proceso de codificación viva es relevante la asignación de una nomenclatura de codificación que respeta la narrativa original expresada por el sujeto (figura 1) (Charmaz, 2004). Una vez seleccionadas las unidades de significado se realiza la codificación axial basada en el tratamiento de las pre-categorías (figura 2) y su integración en nodos emergentes que constituyen las categorías de estudio. 


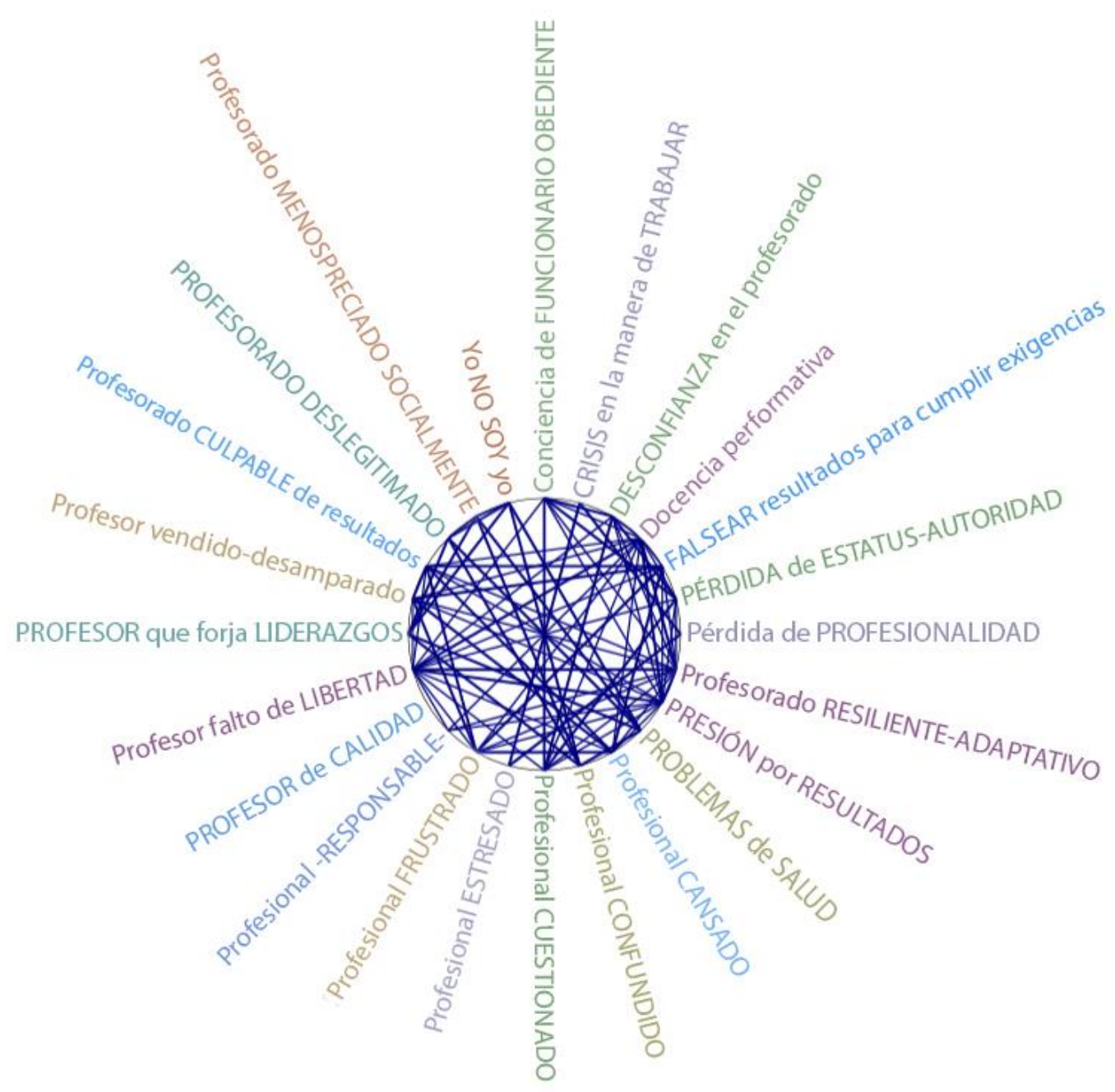

Figura 1. Elementos conglomerados por similitud de palabra

Fuente: Elaborado a partir de QSR NVivo12.

El proceso de análisis prosigue con una fase caracterizada por la tarea de "bricolaje del investigador", fundamentada en integrar los nodos textuales en categorías más amplias. Como se muestra en la figura 2 , aparecen cuatro grandes bloques categoriales que conforman los resultados. En esta fase se estudian las relaciones entre las agrupaciones de nodos y se nombran los bloques categoriales desde las interpretaciones de los investigadores. El primer bloque categorial se denomina "identidades resilientes" y se compone de las pre-categorías: "problemas de salud", "falsear resultados para cumplir exigencias", "conciencia de funcionario-obediente" y "profesorado resiliente-adaptativo". El segundo bloque categorial se entiende como "docentes performativos", que comprende las pre-categorías: "profesor vendido-desamparado", "profesor falto de libertad", "presión por resultados", "profesorado culpable de resultados", "profesor que forja liderazgos", "profesional responsable" y "profesor de calidad". El tercer bloque categorial se denomina "cuestionamiento docente" y se compone de las pre-categorías: "pérdida de profesionalidad", "profesorado deslegitimado", "profesorado deslegitimado socialmente", "desconfianza en el profesorado", "profesional cuestionado", "crisis en la manera de trabajar" y "profesorado estresado". El cuarto bloque categorial, denominado "malestar y 
desmoralización", comprende las pre-categorías: "profesional cansado", "docencia performativa", "profesional confundido", "profesional frustrado" y "yo no soy yo". Para la presentación de resultados se realizan matrices de codificación que relacionan unidades de significado intra-bloque e inter-bloque al objeto de facilitar el hilo conector de los resultados.

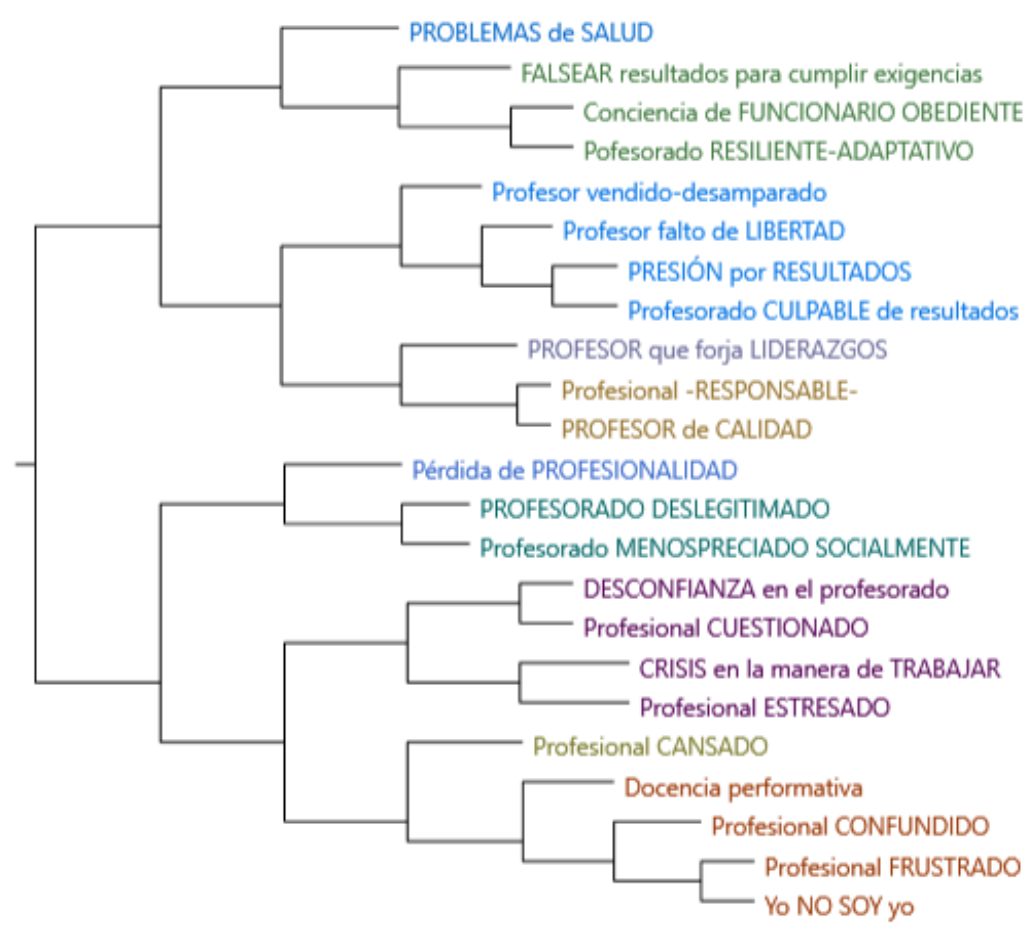

Figura 2. Árbol de nodos resultante de la codificación selectiva Fuente: Elaborado a partir de QSR NVivo 12.

\section{Resultados}

Se decide construir, siguiendo las aportaciones de Sugrue (2015), un espacio teórico que permita comprender el objeto de estudio a través de la fusión de las narrativas de los participantes, las teorías de los investigadores y las evidencias existentes en la literatura académica. Con ello se pretende abrir la investigación para indagar en los posicionamientos onto-epistemológicos sobre los que se construye la teoría. Se trata de una forma de articular la discusión, siguiendo la propuesta de la Teoría Fundamentada, basada en la conjugación de las narrativas de los participantes (emic) con la perspectiva de los investigadores (etic).

En los siguientes subapartados se desarrollan los bloques categoriales. En el primer subapartado de los resultados se desarrolla el bloque categorial denominado "Docentes Performativos"; en cuyas pre-categorías convergen unidades de significado referentes al control sobre el profesorado en un escenario caracterizado por prácticas de evaluación que terminan siendo el fin de la tarea docente y la principal preocupación del profesorado. Esta dimensión presenta frecuentes relaciones con el bloque categorial "Cuestionamiento Docente”; en cuyas pre-categorías se denuncia una merma de la autonomía profesional y una deslegitimación administrativa y social de su práctica que deviene en una pérdida del 
control sobre los quehaceres, ritmos y objetivos del proceso educativo. En el segundo subapartado de los resultados se expone el bloque categorial denominado "Identidades Resilientes"; comprendido por una serie de pre-categorías que hacen referencia a nuevos reclamos profesionales para conformar un sujeto flexible y obediente ante los requerimientos de eficiencia y rendimiento que marca la agenda educativa neoliberal. Este bloque categorial establece asociaciones frecuentes con el bloque "Malestar y Desmoralización"; compuesto por pre-categorías que manifiestan que el profesorado se encuentra desorientado y en el centro de un cuestionamiento permanente que condiciona su ejercicio profesional. A continuación, se desarrollan en profundidad estas cuestiones.

\subsection{La experiencia performativa condiciona la profesionalidad docente}

La cultura del rendimiento se erige como una deidad que impregna diversas esferas personales y profesionales. En educación empieza a no importar tanto las condiciones del alumnado o las situaciones profesionales y personales del profesorado porque lo que interesa son los resultados de los centros y las estadísticas que otorgan valor en escenarios caracterizados por prácticas competitivas y rendición de cuentas (Fuller, 2019):

\section{La exigencia de resultados es una presión mediática, social, de la delegación y de todos los sitios que, en definitiva, no deja que esto sea un espacio para que podamos trabajar a gusto y el profesor pueda considerar al alumno como se merece. (Dmpe4)}

Se está demandando al profesorado que priorice el dictamen evaluativo, los informes de evaluación, las reseñas y las mediciones cuantitativas. Como señalan Saura y Bolívar (2019), las prácticas neoliberales consolidan mecanismos de control, evaluación y comparación que instan a los docentes a centrar su tarea en aquellos aspectos conmensurables que determinan el éxito individual y el bienestar de la organización. La identidad profesional del profesorado se transforma bajo la lógica de la cuantificación, quedando presa del cálculo y la evaluación, aspectos en los que la mayoría del profesorado no se reconoce y que fomentan un desequilibrio de las dimensiones que conforman su identidad profesional.

No sé si utilizar la expresión; pero te sientes un poco vendido o desamparado. Mi madre y mi padre eran maestros y ellos nunca vivieron situaciones de auténtico delirio como las que estamos viviendo nosotros. (Dmzr2)

En la experiencia performativa impera cuantificar, clasificar y datar, lo que genera nuevas formas de organizar y entender la práctica docente. En este caso, se evidencia la fortaleza de una cultura basada en controlar todo lo que desarrolla el profesorado, para lo que se le apremia a tomar mediciones de cualquier situación que ocurra en el aula:

\footnotetext{
To soy profesor, pero necesito un secretario o secretaria porque necesitamos estar tomando continuamente anotaciones de todo, entonces: ¿̨quién da clase? ¿Cómo se da clase? Si yo doy clase y estoy valorando miles de cosas mientras estoy dando clase... tengo que optar por tomar nota o dar clase. (Dmgzr 12)
}

Lo cierto es que, en condiciones de evaluación y control administrativo, las prácticas de endoprivatización limitan la autonomía del profesorado, obviando su juicio experto sobre lo que se enseña y desdeñando su participación en el debate político de la educación (Olivant, 2015):

Cuando practico mi autonomía, la administración me pone en tela de juicio; entonces, ¿̇en qué quedamos? "Quiero resultados" pero, al mismo tiempo, "quiero que seas autónomo", "pero no lo hagas tampoco porque entonces..." La autonomía no existe, es solo una palabra que está ahi y queda muy bien de cara al exterior. (Dmtpe7) 
Se demuestra, en coherencia con lo estudiado por Hendrikx (2019), que la agenda neoliberal media entre las convicciones profesionales de los docentes y aquello que hacen como consecuencia de las prácticas de endoprivatización. Incluso los principios éticos pueden quedar en un segundo plano cuando se genera la necesidad de ser un sujeto eficaz a las demandas performativas, o como lo expresa Ball (2003, p. 92), "la eficiencia prima sobre la ética”:

En el fondo, todos, todos o, al menos, la mayor parte de los profesores falsean los resultados; estoy convencido. (Dhzr5)

Como defienden Sugrue y Mertkan (2017) esto no solo evidencia el fracaso de las políticas de presión y rendición de cuentas basadas en rendimiento académico, sino que empobrece el sentido de responsabilidad profesional y limita el desarrollo docente, que es clave para favorecer discursos y prácticas alternativas en relación al futuro de la profesión y de la calidad educativa. De forma paralela, estas prácticas rompen con el profesorado como agente social crítico.

Yo sí siento esa falta de libertad, esa presión, que a mí me parece más propia de una empresa, en la que hay que obedecer y callar. (Dtszr9)

Podría considerarse que está en disputa el espacio que conforma la profesionalidad de los docentes. Su trabajo queda definido, cada vez más, por instancias ajenas a sí mismos y pierden el control sobre las tareas, tiempos y finalidades de la realidad educativa (Venegas, Usategui y del Valle, 2017):

$$
\begin{aligned}
& \text { Antes éramos médicos y ahora ya nos estamos transformando en enfermeros y lo único } \\
& \text { que hacemos es aplicar la receta que nos da la delegación. (Dmzr2) }
\end{aligned}
$$

Si bien el profesionalismo, en su sentido clásico, es un fenómeno regulatorio donde los profesionales controlan su propia práctica basándose en el conocimiento experto y en la experiencia (Noordegraaf, 2016), la agenda neoliberal quiebra esta condición:

To tengo muchas veces la sensación de: ¿̇qué hago yo aqui? ¿QQué pinto yo aqui? ¿Tengo capacidad de decisión? Ninguna. (Dhpe3)

Son narrativas que reflejan una falta de reconocimiento y una limitación del sentido de competencia profesional, que a su vez influyen sobre su autoimagen. Se percibe que el profesorado se encuentra confundido e inmerso en una incertidumbre profesional que dificulta la estabilidad de la identidad profesional.

Por un lado, te están pidiendo estadísticas; $y$, por otro lado, te están pidiendo alumnos muy buenos para que PISA salga estupendo. Pero no suspendas a alumnos porque entonces los porcentajes de aprobados ya tal. (Dmpe4)

Puede considerarse que la cultura performativa ocasiona serios desajustes en la forma de entender el trabajo como consecuencia del estrés al que están sometidos los docentes:

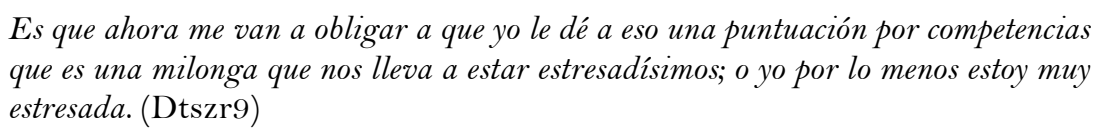

En estas narrativas descansa una de las finalidades de la performatividad, como se señala en Luengo y Saura (2013), que radica en construir sobre los docentes un estado psicológico de inestabilidad emocional e inseguridad en sus decisiones. Estas situaciones ocasionan dolencias físicas o problemas de salud psicológica:

Tienes ganas de llegar a casa, evadirte, disfrutar con los tuyos, pero a veces, toda la presión que acumulas durante el día... pues que si ansiedad, si un dolor de cabeza, una contractura... es agotador. (Dfic 11 ) 
Son hechos que han sido identificados en el ámbito español y en otros casos en el contexto internacional, donde se han estudiado las respuestas afectivas y psicosomáticas que la cultura performativa genera. Se advierten estados depresivos, angustiosos, ansiosos, de incertidumbre y ambivalencia (Singh, 2018). Y es que, las políticas de endoprivatización constituyen un espacio de trabajo caracterizado por la desconfianza en la práctica del profesorado y el enjuiciamiento continuo de la profesionalidad docente:

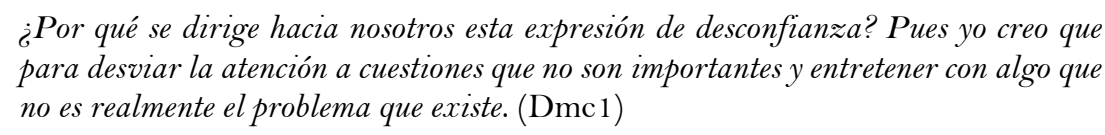

Los docentes se sienten señalados como principales responsables de los problemas que conciernen a la educación, lo que perjudica profundamente su percepción sobre el reconocimiento social atribuido a la actividad profesional que desarrollan:

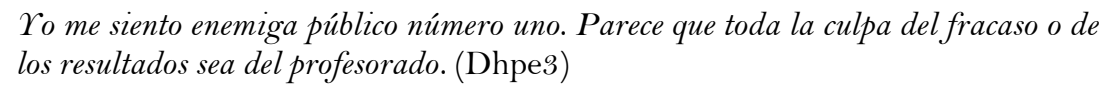

A su vez, esta individualización de la responsabilidad esconde una desviación de la atención que implica dejar de prestar atención a elementos estructurales y condiciones socioeconómicas que explican con más profundidad el desajuste en el logro de los objetivos del sistema educativo.

\subsection{Reconstrucciones "resilientes" de la identidad profesional ante el malestar y cuestionamiento docente}

El contexto descrito deriva en un espacio de desarrollo docente donde la identidad profesional del profesorado se proyecta sobre un escenario incierto, cuestionado y preciso de ser reconstruido (Bolívar, 2006). En este sentido, para mitigar las tensiones de identidad que plantean las prácticas neoliberales de endoprivatización, los docentes intentan reconciliar sus axiomas personales y profesionales con los requerimientos de la cultura del rendimiento. Una parte del profesorado apuesta por adoptar prácticas más seguras, orientadas a que el alumnado obtenga mejores resultados, en un intento de cumplir con las exigencias demandadas (Bailey, 2014):

$$
\begin{aligned}
& \text { Creo que la lógica resultadista en educación ha venido para quedarse y va a ser difícil } \\
& \text { de combatir, por tanto, al profesorado solo le queda adaptarse a los cambios e intentar } \\
& \text { que repercuta lo menos posible en las trayectorias de los estudiantes. (Dgc8) }
\end{aligned}
$$

Se transita hacia una nueva transformación de la docencia que normaliza una identidad profesional alineada a la de un docente performativo (Holloway y Brass, 2018):

$$
\begin{aligned}
& \text { Los centros educativos nos tenemos que mover por una legislación que existe, con unos } \\
& \text { reglamentos, y tenemos que aplicar las cosas tal cual. ¿Eso es calidad? Pues en cierta } \\
& \text { forma sí, porque estoy cumpliendo con lo que me han dicho. (Defc 10) }
\end{aligned}
$$

Es mayoritario un posicionamiento docente que no siempre muestra actitudes de resistencia ante la agenda de la estandarización, el fomento de prácticas mercantiles o los nuevos modelos gerenciales de gobierno, porque resulta menos agresivo para su identidad profesional cumplir con esos cánones (Fuller, 2019).

Quizá esto es un proceso que van a sufrir, antes o después, todas las profesiones. A nosotros nos ha tocado ahora, pero luego pueden ser los médicos, los abogados... Hay cuestiones en la vida que uno no puede controlar, nos gusten más o menos. (Defc10)

Los docentes tratan de integrar en sus esquemas de identidad elementos contradictorios. Significados identitarios procedentes de lógicas profesionales y prácticas propias de la 
endoprivatización para encontrar sentido a su espacio laboral y experimentar conflictos profesionales más livianos que permitan cierta estabilidad y satisfacción con el trabajo (Bévort y Suddaby, 2016).

\begin{abstract}
No es que sea menos combativa que cuando empecé o menos crítica, tampoco creo que mis expectativas e ilusiones como profesora se hayan desvanecido, pero lo que no me apetece es llegar a casa y pagar mi cabreo y mi frustración con mi marido o con mis hijos, entonces tienes que encontrar una vía de escape que te permita mantener un cierto criterio pero que no sea una losa sobre las exigencias que hay que cumplir (Dmpe4)
\end{abstract}

De manera general, no se aprecia en el profesorado un posicionamiento basado en acciones de resistencia profesional (Luengo y Molina-Pérez, 2019). Tampoco un cuestionamiento ontológico que sirva para negociar las tensiones a las que se somete su identidad. Lejos de ello, prevalece un nuevo esquema de identificación, “profesor como funcionario”, que significa una claudicación de los principios y las convicciones pedagógicas ante las demandas exógenas:

\title{
Nosotros somos funcionarios y tenemos conciencia de ser gente que trabaja para hacer que se cumpla la normativa. (Dfc6)
}

Una parte del profesorado se define a través de su relación con la administración más que en su vinculación a una comunidad profesional y educativa. Esto implica la aceptación de un plano secundario en relación a lo que ocurre en su práctica y la constitución de una identidad profesional pragmática y técnica (Luengo y Saura, 2013).

Al final todos intentamos hacer nuestro trabajo porque todos somos profesores $y$ hacemos cada año lo que se nos va diciendo desde delegación. (Dmpe4)

No obstante, sí se detecta en la mayoría de casos un sentimiento de hastío y malestar basado en la evidencia de que una parte del profesorado no se reconoce en su práctica o, al menos, no considera que su práctica sea aquello por lo que lo están reconociendo:

\section{Te encuentras que vienen con una serie de estadísticas y documentos donde tú quedas ahí reflejada. $Y$ yo no soy lo que queda ahí reflejada porque yo creo que a lo largo de los años yo soy lo que el alumno ha ido viviendo conmigo. (Dmtpe7)}

Los docentes se encuentran en la búsqueda de una estabilidad emocional que creen alcanzar cuando aceptan que deben acatar sin cuestionar las reglas de juego. Se asume que su rol es el de sumergirse en los desafíos más que asumir el reto de transformarlos desde un posicionamiento activo.

La resiliencia es una cualidad muy importante para un profesor o un maestro. $Y$ nosotros, al final, nos terminamos adaptando a todo lo que viene. (Dgc8)

La resiliencia, si bien se entiende como "la aptitud para (...) tener el deseo, la determinación y la energía, así como el conocimiento y el fuerte propósito moral que hace que los profesores sean capaces de enseñar de forma óptima” (Day y Gu, 2015, p. 45); se observa que está siendo articulada bajo una notable reinterpretación de la agenda neoliberal (Bailey, 2015):

\footnotetext{
Ahora los temas en el Centro de Formación del Profesorado son el emprendimiento y la resiliencia. "Tenéis que ser resilientes", nos dicen. $\Upsilon$ esas personas que nos dicen eso seguro que nunca han pisado un aula. Uno cuando ya tiene una edad... estas cosas las toma a risa. (Dtszr9)
}

La respuesta docente asociada a la resiliencia trata de normalizar la idea de un profesor que asume de manera absoluta su responsabilidad en la consecución de los objetivos de los 
sistemas educativos y en su bienestar laboral. Por consiguiente, los poderes públicos quedan despojados de su responsabilidad en la consecución de los logros sociales vinculados a la educación. Simultáneamente aleja al profesional del apoyo y respaldo que la administración pública otorgaba a los trabajadores de la educación (Joseph, 2013). Ahora el profesor que se desenvuelve en contextos neoliberales de endoprivatización tiene que adaptarse a los desafíos del presente. Para ello debe ser fuerte, calculador y emocionalmente inteligente para sacar provecho de sus oportunidades ante el entorno competitivo en el que se instala la educación (Bailey, 2015). Se trata de que el profesional no pretenda adoptar un rol crítico comprometido con la justicia social, sino construir su profesionalidad bajo una identidad profesional ágil y moldeable a los requerimientos de las lógicas neoliberales.

\begin{abstract}
Para mí lo normal, sobre todo en las últimas fechas, es la incertidumbre... digamos que ya lo tengo asumido y que me manejo bien en ese escenario. Ahora, por ejemplo, si viene una evaluación, pues preparo la evaluación. Si finalmente no se hace porque se quiere boicotear la LOMCE... pues bueno, sigo con mi planteamiento, esto para mí es lo normal. (Dfic 1 1)
\end{abstract}

La demanda neoliberal de un sujeto flexible y resiliente está siendo interiorizada por el profesorado y se evidencia en su intento por responder con fidelidad a los requerimientos que plantean las prácticas de endoprivatización. Incluso se incorporan estos planteamientos a sus esquemas de responsabilidad:

\title{
Tengo mucho estrés porque soy una persona responsable y quiero intentar cumplir con lo que me mandan. (Dtszr9)
}

Este intento permanente por adaptarse al contexto, mostrarse eficaz bajo cualquier dificultad y cumplir con las exigencias ha sido entendido por O’Malley (2010) como una manifestación de la respuesta resiliente demandada al profesorado. La resiliencia termina erigiéndose como un complejo psicológico constituido por "tecnologías del yo" para optimizar su valor como sujeto (Foucault, 1990). Los profesores, atendiendo a su responsabilidad con la organización, tratan de adaptarse continuamente a las contingencias:

Llega un momento en el que piensas que no puedes más y entonces te adaptas porque: ¡sálvese quien pueda! Es una lucha interna que me produce mayor cansancio.

(Dtszr9)

Esto es coherente con investigaciones recientes que señalan la construcción de nuevas identidades adaptativas como respuesta a las exigencias políticas (Connolly, Milton, Davies y Barrance, 2018). Y es que, la manera de resolver los conflictos de identidad no es a través de respuestas críticas, sino desarrollando identidades resilientes como un medio para mejorar el "yo", para hacerlo más útil en un espacio de demandas volátiles (Bailey, 2015). En consecuencia, el término adopta un nuevo significado en el marco de las reformas neoliberales. El profesor resiliente "debe luchar permanentemente por adaptarse al mundo” (Reid, 2013, p. 355) más que implicarse en procesos de alfabetización política que generen espacios de transformación y compromiso para la mejora de la comunidad.

\section{Reflexiones finales}

Las narrativas de los participantes ponen de manifiesto que las dinámicas de endoprivatización debilitan la autonomía de los docentes, su prestigio y su reconocimiento social. Podría considerarse que actúan como mecanismos para redefinir la identidad 
profesional y generar nuevas formas de pensar el trabajo docente, donde el profesorado es reconfigurado y considerado como un técnico encargado de producir rendimiento académico en el alumnado (Courtney y Gunter, 2019). Este nuevo rol provoca situaciones de malestar y hastío. Se está tratando de tecnificar la docencia y restar al profesorado su espacio de reflexión y crítica. De este modo, se deteriora el compromiso del educador con las finalidades políticas de emancipación y transformación social, porque en la lógica neoliberal prevalece la estandarización, la productividad, la eficacia y la eficiencia.

Las dinámicas de endoprivatización demandan al profesorado una praxis asociada a una experiencia de desempeño, cálculo y medición porque esto es lo que genera valor como profesionales. En consecuencia, la identidad docente se construye bajo una relación que asocia al buen profesor con el docente pragmático que alcanza los objetivos de desempeño por encima de ideales éticos o concepciones de romanticismo profesional. En este contexto, una parte del profesorado manifiesta encontrarse desamparado y confundido ante los intensos cambios que experimenta la profesión docente. Esto redunda en una inestabilidad emocional y personal, además de diversas respuestas afectivas y somáticas, sobre la que se construye su identidad profesional (Luengo y Saura, 2013; Singh, 2018). Se experimentan episodios de reequilibrio de identidad producidos por tensiones entre los juicios profesionales, las demandas performativas y la necesidad de encontrar una estabilidad que mejore la satisfacción profesional.

El éxito de la política educativa se focaliza fundamentalmente en la actitud de los docentes, de modo que adaptarse a los requerimientos es la estrategia para responder con efectividad a las incertidumbres propias de su vida profesional (Bailey, 2014). Es por esto que se persigue una reconstrucción resiliente de la identidad, que no solo implica una adaptación permanente a los desafíos que plantea la realidad social, sino que su valor es importante en la cotidianidad de la práctica docente (Day y Gu, 2015). Esto lleva a considerar que la constante evocación a patrones profesionales como "educador resiliente" modifica severamente el significado del profesional de la educación. Es preciso identificar aquellos discursos que forman parte de la estrategia neoliberal para construir un tipo particular de docente que se adapte a los nuevos reclamos de trabajo eficiente en el contexto de la endoprivatización. En consecuencia, la reconstrucción resiliente de la identidad profesional en el contexto neoliberal insta al profesorado a aceptar un marco de trabajo caracterizado por la desigualdad, la inseguridad y la individualización (Sancho-Gil et al., 2014). A su vez, obliga a los docentes a asumir la responsabilidad individual para su éxito social, económico y profesional. En consecuencia, es necesario tener en cuenta que los nuevos elementos identitarios que propician la adaptación a las demandas de la endoprivatización no son neutrales. Por el contrario, responden a una red de razones complejas que están socialmente situadas y, con frecuencia, influidas por discursos gerenciales hegemónicos que median poderosamente en la definición de la educación y en la naturaleza del trabajo docente.

Para concluir se hace referencia a la necesidad de seguir comprendiendo los acuciantes procesos de cambio que está experimentando el profesorado. Es preciso dar voz a los docentes en investigaciones futuras abordando también técnicas más personalizadas como los biogramas o las historias de vida que permitan adentrarse en aspectos subjetivos más complejos de lograr utilizando la entrevista como instrumento. Así mismo, se precisa abordar trabajos de corte cualitativo que contribuyan a comprender la situación profesional del profesorado para favorecer el éxito de los procesos de reforma. Por ello, desarrollar otros estudios puede aportar a la comunidad científica criterios muy relevantes 
para la calidad de la investigación cualitativa, como la coherencia o la credibilidad asociadas a los procesos de triangulación de informaciones. Otra de las limitaciones de este trabajo es su desarrollo en una única Comunidad Autónoma (Andalucía), por lo que es necesario aportar nuevas investigaciones que puedan informar de otras prácticas e interpretaciones locales, dado la transferencia de competencias en materia educativa del contexto español. De este modo, podrá generarse un espacio proclive a la reflexión, al cuestionamiento y a la crítica de nuevos significantes que pretendan soslayar el sentimiento de hastío y vulneración intelectual del profesorado para construir, al mismo tiempo, espacios de transformación social que han sido limitados por el impulso de la agenda neoliberal.

\section{Referencias}

Bailey, G. (2014). Accountability and the rise of play safe pedagogical practices. Education+ Training, 56(7), 663-674. https://doi.org/10.1108/ET-07-2014-0081

Bailey, P. L. (2015). Consultants of conduct: New actors, new knowledges and new resilient subjectivities in the governing of the teacher. Journal of Educational Administration and History, 47(3), 232-250. https://doi.org/10.1080/00220620.2015.1038696

Ball, S. J. (2003). Profesionalismo, gerencialismo y performatividad. Educación y Pedagogía, 37(15), $87-104$.

Ball, S. J. y Youdell, D. (2008). Hidden privatisation in public education. Bruselas: Education International.

Bévort, F. y Suddaby, R. (2016). Scripting professional identities: How individuals make sense of contradictory institutional logics. Journal of Professions and Organization, 3(1), 17-38. https://doi.org/10.1093/jpo/jov007

Bolívar, A. (2006). La identidad profesional del profesorado de Secundaria. Crisis y reconstrucción. Archidona: Aljibe.

Bolívar, A. y Domingo, J. (2019). La investigación (auto)biográfica en educación. Barcelona: Octaedro.

Bolívar, A., Domingo, J. y Pérez-García, P. (2014). Crisis and reconstruction of teachers' professional identity: The case of secondary school teachers in Spain. The Open Sports Sciences Journal, 7, 106-112. https://doi.org/10.2174/1875399X01407010106

Charmaz, K. (2004). Grounded theory: Objectivist and constructivist methods. En R. M. Emerson (Ed.), Contemporary field research (pp. 109-126). Boston, MA: Little, Brown and Company.

Connolly, M., Milton, E., Davies, A. J. y Barrance, R. (2018). Turning heads: The impact of political reform on the professional role, identity and recruitment of head teachers in Wales. British Educational Research Journal, 44(4), 608-625. https://doi.org/10.1002/berj.3450

Courtney, S. y Gunter, H. (2019). Corporatized fabrications: The methodological challenges of professional biographies at a time of neoliberalisation. En J. Lynch, J. Rowlands, T. Gale y S. Parker (Eds.), Practice methodologies in education research (pp. 27-47). Londres: Routledge. https://doi.org/10.4324/9780429202063-2

Day, C. (2002). School reform and transitions in teacher professionalism and identity. International Journal of Educational Research, 37(8), 677-692. https://doi.org/10.1016/S08830355(03)00065-X 
Day, C. (2018). Professional identity matters: Agency, emotions, and resilience. En P. Schutz, J. Hong y D. Cross (Eds.), Research on Teacher Identity (pp. 61-70). Cham: Springer. https://doi.org/10.1007/978-3-319-93836-3_6

Day, C. y Gu, Q. (2015). Educadores resilientes, escuelas resilientes. Madrid: Narcea Ediciones. https://doi.org/10.32541/recie.2016.v1i1.pp122-123

Denzin, N. K. y Lincoln, Y. S. (2012). Manual de investigación cualitativa. El campo de la investigación cualitativa. Barcelona: Gedisa.

Dubar, C. (2000). La socialisation. Construction des identités sociales et professionnelles. París: Armand Colin.

Flick, U. (2014). El diseño de la investigación cualitativa. Madrid: Morata.

Foucault, M. (1990). Tecnologías del yo. Buenos Aires: Paidós.

Fuller, K. (2019). That would be my red line: An analysis of head teachers' resistance of neoliberal education reforms. Educational Review, 71(1), 31-50.

https://doi.org/10.1080/00131911.2019.1522042

Garner, J. K. y Kaplan, A. (2019). A complex dynamic systems perspective on teacher learning and identity formation: an instrumental case. Teachers and Teaching, 25(1), 7-33. https://doi.org/10.1080/13540602.2018.1533811

Glaser, B. y Strauss, A. (1967). The discovery of grounded theory: Strategies for qualitative research. Nueva York, NY: Aldine de Gruyter. https://doi.org/10.1097/00006199-19680700000014

Gu, Q. y Day, C. (2013). Challenges to teacher resilience: Conditions count. British Educational Research Journal, 39(1), 22-44. https://doi.org/10.1080/01411926.2011.623152

Hendrikx, W. (2019). What we should do Vs what we do: Teachers' professional identity in a context of managerial reform. Educational Studies, 1-17.

https://doi.org/10.1080/03055698.2019.1620694

Holloway, J. y Brass, J. (2018). Making accountable teachers: The terrors and pleasures of performativity. Journal of Education Policy, 33(3), 361-382.

https://doi.org/10.1080/02680939.2017.1372636

Joseph, J. (2013). Resilience as embedded neoliberalism: A governmentality approach. Resilience: International Policies, Practices and Discourses, 1(1), 38-52.

https://doi.org/10.1080/21693293.2013.765741

Kvale, S. (2015). Las entrevistas en investigación cualitativa. Madrid: Morata.

Luengo, J. y Molina-Pérez, J. (2018). (Re)-contextualización del modelo de cuasi-mercado en Andalucía. Dirección escolar y familias compitiendo y seleccionando. Profesorado. Revista de Currículum y Formación de Profesorado, 22(2), 225-243. https://doi.org/10.30827/profesorado.v22i2.7723

Luengo, J. y Molina-Pérez, J. (2019). Construyendo la resistencia profesional en un espacio educativo neoliberalizado. Educatio Siglo XXI, 37(1), 91-112.

https://doi.org/10.6018/educatio.363401

Luengo, J. y Saura, G. (2013). La performatividad en la educación: La construcción del nuevo docente y el nuevo gestor performativo. REICE. Revista Iberoamericana sobre Calidad, Eficacia y Cambio en Educación, 11(3), 139-153. 
Luthar, S. S., Cicchetti, D. y Becker, B. (2000). The construct of resilience: A critical evaluation and guidelines for future work. Child development, $71(3), 543-562$.

https://doi.org/10.1111/1467-8624.00164

Noordegraaf, M. (2016). Reconfiguring professional work: Changing forms of professionalism in public services. Administration \& Society, 48(7), 783-810.

https://doi.org/10.1177/0095399713509242

Noy, C. (2008). Sampling knowledge: The hermeneutics of snowball sampling in qualitative research. International Journal of Social Research Methodology, 1 1(4), 327-344. https://doi.org/10.1080/13645570701401305

Olivant, K. F. (2015). I am not a format: Teachers' experiences with fostering creativity in the era of accountability. Journal of Research in Childhood Education, 29(1), 115-129. https://doi.org/10.1080/02568543.2014.978920

O’Malley, P. (2010). Resilient subjects: Uncertainty, warfare and liberalism. Economy and Society, 39(4), 488-509. https://doi.org/10.1080/03085147.2010.510681

Reid, J. (2013). Interrogating the neoliberal biopolitics of the sustainable development-resilience nexus. International Political Sociology, 7(4), 353-367. https://doi.org/10.1111/ips.12028

Ricoeur, P. (1996). Sí mismo como otro. Madrid: Siglo XXI.

Sancho-Gil, J. M., Hernández-Hernández, F., Martínez-Pérez, S., Padilla-Petry, P., MontanéLópez, A., Herraiz- García, F., ... y Losada-Iglesias, D. (2014). La construcción de la identidad docente del profesorado de educación infantil y primaria en la formación inicial y los primeros años de trabajo. En J. M. Sancho-Gil, J. Correa, X. Giró y L. Fraga (Coords.), Aprender a ser docente en mundo en cambio. Simposio Internacional (pp. 11-23). Barcelona: Dipòsit Digital de la Universitat de Barcelona.

Saura, G. y Bolívar, A. (2019). Sujeto académico neoliberal: Cuantificado, digitalizado y bibliometrificado. REICE. Revista Iberoamericana sobre Calidad, Eficacia y Cambio en Educación, 18(1), 9-26. https://doi.org/10.15366/reice2019.17.4.001

Singh, P. (2018). Performativity, affectivity and pedagogic identities. European Educational Research Journal, 17(4), 489-506. https://doi.org/10.1177/1474904117726181

Smith, J., Flower, P. y Larkin, M. (2009). Interpretative phenomenological analysis: Theory, method and research. Londres: Sage.

Strauss, A. y Corbin, J. (2002). Bases de la investigación cualitativa. Técnicas y procedimientos para desarrollar la teoría fundamentada. Medellín: Universidad de Antioquía.

Sugrue, C. (2015). Unmasking school leadership: A longitudinal life history of school leaders. Dordrecht: Springer. https://doi.org/10.1007/978-94-017-9433-6

Sugrue, C. y Mertkan, S. (2017). Professional responsibility, accountability and performativity among teachers: The leavening influence of CPD? Teachers and Teaching, 23(2), 171-190. https://doi.org/10.1080/13540602.2016.1203771

Vallés, M. S. (2009). Entrevistas cualitativas. Madrid: Centro de Investigaciones Sociológicas.

Venegas, M., Usategui, E. y del Valle, A. I. (2017). El profesorado de secundaria en España ante las políticas de rendición de cuentas en el marco de implantación de la LOMCE. RASE. Revista de Sociología de la Educación, 10(3), 431-454. https://doi.org/10.7203/RASE.10.3.9951

Verger, A. y Normand, R. (2015). Nueva gestión pública y educación: Elementos teóricos y conceptuales para el estudio de un modelo de reforma educativa global. Educação छْ Sociedade, 36(132), 599-622. https://doi.org/10.1590/ESo101-73302015152799 
Waller, M. A. (2001). Resilience in ecosystemic context: Evolution of the concept. American Journal of Orthopsychiatry, 71(3), 290-297. https://doi.org/10.1037/0002-9432.71.3.290

Wengraf, T. (2012). Qualitative research interviewing. Londres: SAGE.

\section{Breve CV de los autores}

\section{Javier Molina-Pérez}

Personal Docente e Investigador en formación con vinculación contractual FPU (Formación de Profesorado Universitario) en el Departamento de Pedagogía de la Universidad de Granada. Graduado en Pedagogía y Máster en Investigación, Desarrollo Social e Intervención Socioeducativa por la misma Universidad. Las líneas de investigación se centran en el ámbito de la política educativa y las publicaciones abordan el análisis de los cambios en la profesión docente, las prácticas de endoprivatización educativa y las transformaciones de la identidad profesional docente. ORCID ID: https://orcid.org/o000-0001-6751-2199. Email: javimp@ugr.es

\section{Julián Luengo}

Profesor Titular de la Universidad de Granada. Director del Departamento de Pedagogía. Doctor en Ciencias de la Educación por la Universidad de Granada. Miembro del grupo de investigación "Valores emergentes, Educación Social y Políticas educativas". Autor de diferentes publicaciones nacionales e internacionales referentes a las principales líneas de investigación que atienden la exclusión social; equidad educativa; políticas y reformas educativas y agendas globales de privatización educativa. Ha participado como investigador en Proyectos financiados por la UE (EGSIE), por la AECID en países latinoamericanos, Plan Nacional $\mathrm{I}+\mathrm{D}+\mathrm{i}$ y Proyectos de Excelencia de la Junta de Andalucía. ORCID ID: https://orcid.org/O000-0001-7015-7415. Email: jluengo@ugr.es 